\title{
O.S.P.
}

L'orientation scolaire et professionnelle

$35 / 1 \mid 2006$

Varia

\section{Projets scolaires, histoires résidentielles et culture locale}

School projects, residential histories and local culture

Jean-Luc Roques

\section{OpenEdition}

1 Journals

Édition électronique

URL : http://journals.openedition.org/osp/914

DOI : $10.4000 /$ osp.914

ISSN : 2104-3795

Éditeur

Institut national d'étude du travail et d'orientation professionnelle (INETOP)

Édition imprimée

Date de publication : 15 mars 2006

Pagination : 83-108

ISSN : 0249-6739

\section{Référence électronique}

Jean-Luc Roques, «Projets scolaires, histoires résidentielles et culture locale », L'orientation scolaire et professionnelle [En ligne], 35/1 | 2006, mis en ligne le 28 septembre 2009, consulté le 04 mai 2019 URL : http://journals.openedition.org/osp/914 ; DOI : 10.4000/osp.914

Ce document a été généré automatiquement le 4 mai 2019.

(c) Tous droits réservés 


\title{
Projets scolaires, histoires résidentielles et culture locale
}

\author{
School projects, residential histories and local culture
}

Jean-Luc Roques

\section{Introduction}

1 Dans le domaine de la sociologie de l'école, les effets du contexte font l'objet depuis plusieurs années de nombreuses recherches. Les études sur l'effet établissement, les travaux sur l'influence de la configuration des offres locales, les enquêtes sur les politiques locales sont là pour en attester. Cet article propose d'étudier, pour sa part, les rapports entre le contexte local, voire la communauté environnante ${ }^{1}$, et les attitudes scolaires de jeunes de classes de troisième de collège.

2 Une hypothèse admise est que les jeunes intégreraient dans leurs parcours les possibilités de formation et détermineraient leurs cursus en conséquence (Huteau, 1992 ; Duru-Bellat \& Van Zanten, 2002). Ils tiendraient compte non de repères qui n'ont plus cours, mais de données concrètes. Les contraintes de l'environnement et les places disponibles affecteraient les conduites (Duru-Bellat, 2002). Bien loin de contredire ces résultats, nous proposerons une orientation complémentaire, qui traitera du local en tant que porteur d'une culture locale spécifique, d'un état d'esprit particulier (Park, 1925) qui véhicule des images, dont l'école n'est pas exempte. Le local est pour le moins une notion générale et abstraite, car on ne sait où il débute et où il se termine. Nous prendrons comme entité de référence et unité concrète la commune. Celle-ci est délimitée par un territoire, possède une autorité légitime, détient un budget particulier et dispose d'un marché scolaire typique. Mais une commune n'est pas que cela. Chacune délimite ce qui est de l'ordre du dedans et ce qui est de l'ordre du dehors (Roncayolo, 1997). Chacune développe à partir d'une entité territoriale une mémoire spécifique et un rapport au sol qui n'est qu'à elle, et construit des représentations originales (Berger \& Roques, 2005). La question sera alors de savoir quels liens existent entre les comportements scolaires des jeunes et cette culture locale? 
3 Dans une première partie, nous présenterons notre méthodologie d'approche dans le cadre d'une étude empirique. Dans une seconde partie, nous verrons la manière dont les jeunes vivent leur scolarité mais aussi leur ville. En guise de conclusion, nous discuterons de la portée de nos résultats.

\section{Présentation de l'étude}

4 Afin de délimiter notre étude, nous avancerons quelques constats, puis nous préciserons notre échantillon, avant d'aborder les trois méthodes d'investigation que nous avons utilisées.

\section{Quelques constats de départ}

5 Le présent travail fait suite à deux études que nous avons menées dans le département du Gard. Une première étude portait sur les attitudes de 350 jeunes de cinq petites villes à l'égard de la santé (Berger, Fosse \& Roques, 1993). Les résultats montraient que les comportements variaient nettement d'une commune à l'autre. Le degré d'investissement dans sa santé, le degré de connaissance des maladies, le désir d'implication dans des actions de santé, étaient différents pour chacune des localités. Par-delà le problème social de la santé chez les jeunes, un problème sociologique émergeait. La dimension spatiale jouait un rôle non négligeable dans les conduites. À partir de ce constat, nous avons posé une nouvelle question relative aux effets du local sur d'autres types d'attitudes. Une seconde étude proposait de comparer les projets scolaires, professionnels et de vie de 733 jeunes de deux petites villes ${ }^{2}$ (Roques, 2004a), mais aussi la manière dont ils représentaient leur espace. Les résultats confirmaient le lien entre conduites juvéniles et lieu de résidence. Les taux de projets, quels qu'ils soient, étaient tous supérieurs dans l'une des deux communes. Afin d'expliquer cette différence nous avons proposé deux hypothèses interprétatives. L'une était centrée sur le différentiel des offres (filières scolaires, emplois, activités diverses). La validité semblait nette puisque dans la ville qui avait le plus d'offres les taux de projets étaient plus importants. Or, les jeunes sont-ils si rationnels que cela? Ont-ils une telle connaissance et telle visibilité des offres? L'autre hypothèse proposait alors de s'intéresser aux représentations et plus particulièrement aux images de l'école, du travail et de la sphère publique qui sont véhiculées localement (Roques, 2003a). Les oppositions entre les deux communes apparaissaient encore bien significatives. Les cultures locales, leurs histoires, leurs mémoires étaient dissemblables et étaient maintenues et entretenues par des groupes locaux fortement intégrés. La construction d'un projet relevait alors de la possibilité d'intégrer l'imaginaire de cette communauté locale et les groupes qui le constituent. Pour vérifier cela, nous avons fait appel aux cartes mentales (point sur lequel nous reviendrons plus loin) qui montraient que les types de projets étaient liés à la manière dont les jeunes représentaient leur ville, à la façon dont ils percevaient les diverses formes sociales dominantes et à la place qu'ils pensaient avoir à l'intérieur de celles-ci.

6 L'orientation de notre travail est donc de valider ce lien entre projet et contexte spatial mais aussi de mettre en perspective la dynamique d'un tel lien. Quels pouvaient être les facteurs qui entrent en jeu dans cette relation? Nous avons alors choisi de réduire encore notre échelle d'analyse, tant en ce qui concerne l'unité concrète étudiée, qu'en ce qui concerne le nombre d'individus, ou en ce qui concerne notre méthode. Nous avons porté 
notamment notre attention sur les projets scolaires, sur les histoires résidentielles, mais aussi sur la représentation de l'espace, de quelques jeunes d'une petite ville du sud de la France.

\section{L'échantillon}

7 La commune étudiée est celle d'Uzès dans le département du Gard. Cette petite ville est entourée d'une zone naturelle ou agricole, et les liaisons routières pour accéder à de plus grandes agglomérations sont modestes. La population y est d'environ 9000 habitants dont 600 jeunes de 15 à 19 ans. En l'absence de toute industrialisation, relativement éloignée des centres décisionnaires, c'est une ville à dominante touristique qui est protégée et bénéficie d'avantages pour l'amélioration du patrimoine. Le passé y est très présent et s'impose (Park, op. cit.) à travers ses rues, ses bâtiments anciens et surtout son duché. Elle s'est chargée de topographies imaginaires (Namer,1987) par le biais de ses monuments et de ses histoires. La ville s'incarne avec des figures emblématiques comme Racine, la famille Gide, mais aussi la lignée ducale, et perpétue une tradition centrée sur la culture architecturale, sur les arts et sur les lettres. De par ce relatif isolement, cette densité de population faible, et cette scène particulière, il existe une culture locale particulière (Durkheim, 1893 ; Halbwachs, 1938). En effet, cette ville n'a pas simplement une existence objective, elle est une construction collective (Van Zanten, Payet \& Roulleau-Berger, 1994). Elle se vit comme une communauté tournée vers son passé, dominée par une certaine forme d'enracinement (Roques, 2004b). Il se dégage de ces lieux, un sentiment d'appartenance territorial fort, un moyen d'identification et une expression identitaire (Kaiser, 1990, p. 250), une culture locale qui allie à la fois sentiment d'une communauté et sens d'une territorialité (Lewis, 1963). L'idéal du passé projette les individus vers autrefois. Le noyau qui maintient et diffuse cet esprit local (Halbwachs, 1925) est constitué par les retraités, les personnes dont l'activité est liée au bâti, les Protestants, les artistes, « les lettrés ». Pensons à cette exposition récente, après l'achat par la médiathèque d'un bréviaire (suite à un appel à don) datant de 1472 écrit à Uzès, intitulée : «Un livre, une foi, une ville ». Or, s'il existe une culture locale particulière quelle place laisse-t-elle aux jeunes? L'enracinement et le sentiment d'appartenance influencent-ils les conduites juvéniles?

Comme dans beaucoup de petites villes isolées, le nombre d'établissements scolaires est limité. Il existait deux collèges ${ }^{3}$, au moment de notre étude, le collège privé «Saint Firmin » et le collège public « Lou Redounet ». Les lycées sont au nombre de trois. Le lycée Charles Gide offre un type de formation générale. Son origine est ancienne, car dès le XVI e siècle, il y avait un régent, des maîtres enseignaient le grec, le latin, les sciences et l'Académie d'Uzès avait le droit de délivrer des diplômes. Créé officiellement en 1837, il est situé dans un bâtiment de style empire, avec sa grande cour intérieure. Son existence fut menacée en 1974 par des mesures administratives, mais un Comité de défense permit d'aboutir à son maintien définitif. Cette mobilisation, présente dans la mémoire collective locale (Halbwachs, 1950), reposait plus sur le maintien de dynamiques culturelles spécifiques que sur des considérations pédagogiques (Duru-Bellat \& Van Zanten, op. cit, p. 95). La noblesse de la filière classique dans cette ville s'inscrit dans une sorte d'idéal humaniste. Le lycée Guynemer propose, pour sa part, un enseignement professionnel, mais le recrutement des élèves se fait paradoxalement peu sur la commune. De plus, les autorités locales ont voulu donner une autre image à cet établissement qui est devenu 
dans le courant des années 1990 : «L.P. des métiers d'Arts ». Il faut ajouter à ces deux établissements, celui du Grand Mas qui dépend des Maisons familiales et rurales d'éducation et d'orientation. Ce dernier a des classes agricoles. Situé à l'extérieur de la ville, il est installé dans une ancienne demeure entourée d'un grand parc. Les filières scolaires locales sont donc réduites, notamment dans le domaine professionnel. Dans ce contexte, les élèves de troisième de collège ne sont pas tous affectés dans des filières de la localité, ce qui oblige une partie d'entre eux à aller dans des villes voisines. Quelles sont dès lors les conséquences sur les conduites des jeunes?

Nous avons choisi d'étudier un échantillon de collégiens de classe de troisième. À la différence des études précédentes, il y a ici peu d'exhaustivité statistique dans l'échantillon délimité. Ce travail s'appuie sur 30 élèves, dont la tranche d'âge variait de 13 à 17 ans. Nous avons rencontré 14 jeunes au collège "Saint Firmin", et 16 jeunes au collège «Lou Redounet ». Ces collégiens ont été choisis en fonction de 3 caractéristiques : le sexe, l'âge et le lieu d'habitation dans la commune. Pour le sexe, nous avons sélectionné de manière quasi équivalente 14 garçons et 16 filles. En ce qui concerne l'âge, nous avons pris autant de jeunes de 14 ans et de 15 ans, soit 10 et 11 , dans une proportion qui correspond à un tiers de l'échantillon, quelques jeunes de 16 ans, soit 5 , et peu d'élèves des catégories extrêmes de 13 ans et 17 ans, soit 1 et 2 . Enfin, pour le lieu d'habitation, notre option était de ne traiter que des jeunes résidant dans la commune. Il est à noter que la ville ancienne est en forme de disque. Elle est entourée d'un boulevard qui correspond aux anciens murs d'enceinte. Ce premier espace est ce que nous appellerons le «centre de la cité ». Au-delà de cette limite, d'anciennes bâtisses collectives, d'échoppes et de maisons plus récentes, composent un « $1^{\text {er }}$ cercle ». À la suite de cette seconde aire et dans ce même jeu de circularité, on voit apparaître plusieurs blocs collectifs de type «H.L.M.» qui forment un regroupement d'habitations. Enfin, un peu à l'écart vient une quatrième zone "extérieure " qui est plus naturelle avec des mas et résidences isolés. Pour répartir notre population d'élèves en fonction de ces quatre zones, et ce qui nous paraissait relever de la répartition globale, nous avons pris 6 jeunes du " centre de la cité », 12 jeunes dans le « $1^{\text {er }}$ cercle », 8 jeunes dans les quartiers « H.L.M. » et 4 jeunes « extérieurs».

Pour appréhender la ou les relations entre les attitudes scolaires et le contexte spatial, nous avons utilisé trois techniques particulières: un questionnaire, des dessins et un entretien non-directif. Voyons ce que recouvrent ces différentes méthodes d'approches.

\section{Projet scolaire et échelle d'attitude}

11 L'école est devenue aujourd'hui un lieu important de l'expérience juvénile (Dubet, 2004), un lieu de relation aux autres, un lieu où l'on change de classe, de filière, un lieu enfin où l'on est amené à passer des diplômes. L'entrée se fait de plus en plus tôt et la sortie se réalise de plus en plus tard. Dans cette situation, une question est posée de manière récurrente aux élèves, qu'elle vienne des parents et des proches, des équipes pédagogiques, ou même des pairs: "Que veux-tu faire, l'an prochain, dans deux ans, dans trois ans... ? ". Question d'autant plus sensible, à la fin de la classe de troisième, que l'élève se trouve dans l'obligation de se doter d'un projet (Dumora, 1990). Cette injonction au projet (Biemar, Philippe \& Romainville, 2003), encore plus problématique pour ceux qui ont peu de ressources, attend une réponse précise, un objectif défini et des moyens à mettre en œuvre conscients. Comme si les choix se faisaient selon un simple processus 
linéaire, alors que l'on sait que ce n'est bien souvent pas le cas (Dumora, 1992). De plus, qui peut prétendre répondre à une telle question tant l'avenir est flou (difficulté de linéarité dans les études, lien étroit «toutes choses égales part ailleurs » entre étude et emploi), tant les différences territoriales sont importantes (filières implantées dans certaines zones et pas dans d'autres), tant la visibilité et la lisibilité des filières est complexe (multiplicité des filières, des options, finalités incertaines). Comment dès lors les jeunes peuvent-ils se construire dans un tel labyrinthe?

Le terme de labyrinthe n'est pas utilisé de manière fortuite, puisque son étymologie renvoie au travail sur soi (labor-intus). Ainsi l'individu est amené à se construire dans cette recherche de singularité, par et dans un segment social temporel et spatial qui est l'école. Ne parle-t-on pas d'ailleurs du métier d'élève, de carrière scolaire, de travail scolaire, de compétences, comme s'il existait un net parallélisme entre la position sociale des élèves et celles des travailleurs (Dubet, 1973). Nous faisons donc le pari d'utiliser le concept de projet de mobilité, lié à l'origine à la sociologie du travail, dans le champ de l'éducation, et cela pour deux raisons. Tout d'abord, ce choix découle de ce qu'il intègre une dimension créatrice pour l'acteur à l'intérieur d'une institution particulière. En effet, le projet recouvre la notion d'action et semble plus riche, à notre sens que d'autres variables comme celle de choix professionnel. Mais si le projet, comme nous l'entendrons, agglomère bien des dimensions (autonomie, expérience, représentation) et apporte une dynamique propre aux acteurs, donner une capacité créatrice à tous les élèves reste une gageure; gageure théorique qui s'oppose à la seule reproduction, gageure méthodologique qui donne la parole aux élèves. Ensuite, le projet de mobilité admet le changement statutaire interne à la situation scolaire. Ce changement est plus sensible dans le passage au collège, puis au lycée, puis vers l'enseignement supérieur. Nous ne parlerons donc pas d'orientation vers un emploi, un travail, un métier car cela relèverait du projet professionnel. Nous ne traiterons pas non plus du passage inter-statutaire entre enfance et âge adulte, tant il est de plus en plus difficile de trouver un emploi, de construire une famille ou d'obtenir un logement autonome (Galland, 1991), mais aussi tant les critères pour définir l'adulte semblent incertains (Ehrenberg, 1995).

13 Nous concentrerons notre analyse sur le projet de mobilité en tant que système d'orientation significatif à l'égard d'une situation socialement et culturellement construite qui est la scolarité. Nous parlerons dès lors de projet scolaire en tant que système d'attitude et de comportement, à l'égard de la situation scolaire, tendu entre deux extrêmes: la participation et le retrait. Nous ne traitons pas de réussite ou d'échec mais plutôt d'attitudes entre deux pôles. La participation s'oriente vers une intention active de mobilité et le retrait indiquera un faible niveau d'implication (Bassoul, Bernard \& Touraine, 1960). De là, nous avons construit une typologie de projets qui a fait l'objet de travaux antérieurs (Roques, 2003a, 2004a). Elle nous servira de base méthodologique, mais aussi d'hypothèse. L'objectif est donc de repérer les diverses attitudes des élèves à l'égard d'une situation particulière, sans entrer dans le débat sur le lien entre projet et réussite scolaire, tant celui-ci pose problème (Bernet, 2000). Dans le cas où l'élève est participatif, nous dirons qu'il est « en projet » avec la situation scolaire. Dans le cas où il est en retrait, nous admettrons qu'il est "en anti-projet». Ici, ce n'est pas de culture anti-école qu'il s'agit, mais d'une simple situation dans laquelle l'élève ne se reconnaît pas et peut envisager d'autres perspectives qu'elles soient réalistes ou utopiques. Entre les deux extrêmes, l'élève sera considéré comme étant «en projet médian ». Sa vision de l'école sera 
bipolaire, tendue entre désir de reconnaissance et désillusion. Le projet représente donc une activité et en aucun cas un simple désir ou un souhait (Dubet, 1991 ; Guichard, 1995).

Rappelons rapidement comment nous avons construit cette échelle d'attitude ${ }^{4}$. Pour définir les niveaux de projets, nous tenons compte de trois dimensions: le degré d'autonomie des individus, le vécu scolaire au quotidien, les représentations d'avenir. La scolarité est, en France, obligatoire jusqu'à 16 ans. Mais au-delà, les jeunes sont pour la plupart à l'école. Nous dirons que la situation scolaire oblige, de fait, les élèves à faire des choix. Or, quelle est la véritable autonomie desjeunes dans ce processus? Cinq indicateurs mesurent ce degré de liberté de choix scolaire (autonomie dans les choix scolaires, autonomie dans le choix d'être scolarisé, autonomie quotidienne, autonomie dans le travail scolaire, autonomie dans les choix d'orientation). Les jeunes vivent aussi une expérience quotidienne (Dubet, 1994 ; Rochex, 1995) dont le cadre est l'établissement scolaire. La vie, à l'intérieur des écoles, est plus importante qu'on ne l'a longtemps pensé (Duru-Bellat \& Mingat, 1988 ; Derouet, 1988 ; Dubet, Cousin \& Guillemet, 1989) et le temps passé à l'intérieur est significativement important (Zaffran, 2000). Dans ce contexte comment les jeunes vivent-ils cette expérience? Cinq indicateurs permettent de rendre compte du vécu scolaire (satisfaction à l'égard de l'établissement fréquenté, satisfaction à l'égard de la scolarité, satisfaction à l'égard de la classe, sens de la réussite scolaire, type d'apprentissage à l'école). Enfin, cette situation entraîne la construction d'images de futures filières. Si les jeunes ont généralement une bonne opinion de leur école, quelles images ont-ils de leur avenir scolaire ? Cinq indicateurs estiment cette représentation de l'avenir scolaire (durée des études envisagée, orientation envisagée à court terme, orientation envisagée à long terme, représentation du lien entre l'école et le travail, perception de l'utilité de l'école). réponses sont proposées et un seul choix est possible. Les deux premières réponses (codées 1 et 2) représentent une attitude plutôt participative. Les deux autres (codées 3 et 4) correspondent à une attitude plutôt de retrait. Par exemple, la question 4 est formulée de la façon suivante : "Que fais-tu lorsque tu as des devoirs à faire après l'école?». Les réponses sont: « Tu les prépares seul et immédiatement (codé 1), tu les prépares bien que ça t'énerve (codé 2), tu attends un peu (codé 3), tu attends que quelqu'un te l'impose (codé 4) ». La résultante des 15 réponses permet de construire un indice de participation et de classer les individus par niveaux de projet. Certes ce type de classification pose un problème méthodologique (Javeau, 1992, p. 87) et c'est en maintenant une certaine retenue que nous abordons ces attitudes. Ainsi, de 10 à 15 réponses, codées 1 ou 2, les élèves sont considérés comme étant «en projet ». De 7 à 9 réponses, codées 1 ou 2, ils sont définis comme étant "en projet médian ». Enfin de 0 à 6 réponses, codées 1 ou 2 , les jeunes sont circonscrits comme étant "en anti-projet». Il est à préciser que ces types d'attitudes représentent des tendances et en aucun cas des conduites totalement rigides. Il existe de nombreux basculements et de nettes variations des conduites (Roques, 2003b).

\section{Histoires résidentielles et représentations de l'espace}

16 À la suite de la passation du questionnaire, nous avons utilisé deux approches particulières afin d'appréhender les rapports qu'entretiennent les collégiens avec leur territoire urbain. La première renvoie aux images que les jeunes ont de leur ville et à leurs représentations de l'espace. La seconde s'inscrit dans les discours qu'ils ont à l'égard 
de la communauté environnante et du territoire dans lequel ils vivent. Nous avons rencontré ces élèves pendant environ une heure à l'intérieur des établissements scolaires, souvent lors d'un de leur cours ce qui limitait le temps de l'entretien.

Nous sommes partis tout d'abord de la représentation que les jeunes avaient de leur ville, et notamment des plans de celle-ci que nous leur avons demandé de dessiner. Les habitants, en effet, ne sont pas neutres et les images de la ville se développent à partir de leur expérience, mais aussi de leur mémoire et de leur souvenir (Lynch, 1960). L'utilisation des cartes mentales introduite par Lynch dans les années 1960, renvoie donc aux expériences individuelles, et l'apparence d'une ville donne lieu à des perceptions différentes selon les personnes qui y vivent. Or, si chacun porte en lui une image typique, l'hypothèse est qu'il existe une concordance entre l'individu et les membres de son groupe d'appartenance. Par ces représentations graphiques, nous avons porté notre attention aux langages spatiaux, qui envisagent de saisir certaines productions du monde physique mais aussi social (Ostrowetsky, 1996). Cela permettait de rendre compte de l'image que l'individu attribuait à son espace et du sens qu'il pouvait lui donner. En utilisant les cartes mentales, nous supposions que le rapport à la ville était une composante importante de l'expérience juvénile (Felloneau, 1994).

Certains de nos travaux précédents inspirés de recherches portant sur des étudiants (Peron, 1994), avaient pointé des différences de représentation entre diverses villes pour des populations équivalentes. Nous avions déjà utilisé cette technique et les résultats montraient qu'il existait une relation nette entre les formes spatiales et les attitudes juvéniles (Roques, 2004b). Le but était de confirmer cette liaison. Nous avions répertorié 5 formes de plans dont les significations étaient différentes. La ville circulaire avait l'image $\mathrm{du}$ rond. Cela représentait plus particulièrement la fermeture territoriale et le soutien qu'apportait la communauté englobante. La ville circulation avec ses voies de communication faisait penser à un réseau. Elle connotait la mobilité, le déplacement et l'ouverture vers l'extérieur. La cité devenait un lieu de passage. La ville réduite proposait l'image d'une forme isolée, comme une maison, ou un lieu particulier. Cette représentation de l'espace était corrélative d'une situation d'isolement de l'individu qui n'appartenait à aucun groupe. La ville éclatée offrait l'image de plusieurs lieux, de divers bâtiments, sans qu'il y ait de lien entre eux. Il y avait ici une absence de cohésion entre ceux-ci. Une dernière figure marginale que nous avions appelée indéterminée n'entrait dans aucun des types précédents (nous n'en parlerons pas ici). La conceptualisation de l'espace (Stoetzel, 1963) est bien différente dans les différents cas. Une petite ville en tant qu'objet spatial est bien différente d'une métropole. Sa taille réduite peut faire penser que les observateurs peuvent connaître rapidement l'ensemble de cette structure spatiale. Les représentations seraient alors toutes similaires. Or, pourquoi les cartes mentales recèlent des divergences? Pourquoi existe-t-il des organisations, des sélections particulières ? S'orienter dans une ville n'est alors pas simplement lié à sa capacité de lire un plan et à se déplacer. Ne faut-il pas voir dans la possibilité, ou la capacité, que certains individus ont de décoder l'état d'esprit des lieux et la culture locale d'autres facteurs qui facilitent l'orientation?

19 Après avoir précisé, la direction générale de la recherche, nous avons demandé aux jeunes de notre échantillon de "dépeindre", en quelques traits, leur ville. La consigne était simple: "Dessine moi ta ville». Un simple carré délimitait le cadre à l'intérieur duquel ils dessinaient leur espace de vie. Pour décrypter les différents dessins, nous nous sommes d'abord attaché à la forme d'ensemble que nous avons précisé plus 
avant et que nous avons classé. Ensuite, nous avons utilisé trois des cinq variables que Lynch avait mis en évidence (Lynch, op. cit., p. 54-55) : les voies, les limites et les points de repère. Les voies sont des lignes de déplacement comme les rues. Les limites sont des frontières entre deux espaces. Pour les points de repère, nous avons surtout tenu compte des éléments du patrimoine local et des établissements scolaires qui pouvaient être dessinés. L'hypothèse était que nous devions retrouver 4 formes de plans et notamment dans cette ville une sureprésentation de "ville circulaire » et de "ville éclatée ». Nous supposions qu'il existait en effet deux grandes catégories de jeunes. Ceux qui font référence aux rues, construisent une frontière nette de leur territoire, représentent des éléments du patrimoine et les établissements scolaires. Ceux qui ne font pas appel aux voies de circulation et délimitent une multiplicité de petits territoires, et ne dessinent ni les espaces patrimoniaux, ni les lieux scolaires.

20 À la suite de cet exercice, nous avons réalisé avec chaque élève un entretien non directif, ou peu structuré. Nous avons utilisé quelques éléments de "récits de vie» (Bertaux, 2005) des jeunes rencontrés et notamment leur histoire résidentielle. Ce qui nous intéressait plus particulièrement était le rapport qui peut exister entre le vécu individuel, l'expérience quotidienne et la culture locale (Thomas \& Znaniecki, 1919). La phrase d'entrée était systématiquement la même: «Parle-moi de ta ville». Dans cette logique de type exploratoire, il était laissé aux jeunes la liberté de leur argumentation et de leur explication. Nous ne faisions que relancer les propos en reprenant les points qui nous semblaient importants pour saisir les éléments qui reliaient ou qui éloignaient ces jeunes de ce local. Nous ne faisions que relancer afin d'approfondir quelques axes. Plusieurs questionnements recouvraient ces entretiens, mais un fil conducteur définissait la direction : la manière dont ils vivaient et avaient vécu cette ville.

21 Notre grille d'analyse s'est calée sur ce thème principal. Les thématiques secondaires et les spécifications qui nous interpellaient dans ces récits étaient le sentiment d'appartenance des jeunes à l'égard du territoire et de la communauté locale. Se sentaient-ils enracinés dans cet espace et dans cette culture des lieux? L'idée était de relever si ce sentiment d'appartenance semblait plutôt positif ou plutôt négatif, s'ils aimaient leur ville, s'ils désiraient y rester, s'ils se sentaient attachés à cette cité. Mais aussi comme l'école est un lieu majeur de l'expérience juvénile, il nous paraissait opportun de connaître leur perception de la scolarité et plus particulièrement la manière dont ils voyaient les établissements scolaires locaux. Quelles images avaient-ils des lycées de la ville? Quelles étaient les personnes qui leur apportaient des informations sur ces établissements? L'école participait-elle du sentiment d'appartenance? Ensuite, nous voulions savoir comment ils ressentaient l'état d'esprit de la ville. Pensaient-ils qu'il existait une communauté homogène ou si celle-ci était plutôt fragmentée en plusieurs groupements? Mais aussi comment ils se situaient eux-mêmes au sein de cette culture locale. Se sentaient-ils intégrés ou plutôt exclus? De la même manière que précédemment, l'école participait-elle à l'intégration ou à la disqualification des individus? Notre intention n'était pas de faire une analyse sémantique, mais plus simplement d'élaborer une typologie à partir de ces deux variables que sont le sentiment d'appartenance et la perception de la communauté locale. Notre hypothèse était qu'il devait exister deux grands types de conduite. Les enracinés auraient un sentiment d'appartenance fort à la cité, à l'histoire, percevraient la communauté locale comme nettement intégrée et homogène. Les établissements scolaires seraient ici considérés de façon positive. À l'opposé les non enracinés auraient un faible sentiment d'appartenance, 
verraient la communauté locale de manière fragmentée et porteraient un jugement négatif sur les établissements scolaires.

\section{Niveaux de projet et contexte spatial}

Pour mettre en évidence les rapports entre les attitudes scolaires et le contexte spatial, notre hypothèse générale était que les niveaux de projets seraient en interaction avec le sentiment d'appartenance et d'enracinement, mais aussi la manière de représenter sa ville. Les jeunes "en projet » se sentiraient enracinés et soucieux de représenter une cité qui les englobe. Les jeunes «en anti-projet» n'auraient qu'un faible sentiment d'appartenance et verraient cette ville comme incohérente et éclatée. Les jeunes «en projet médian" seraient dans une situation intermédiaire, oscillant entre diverses attitudes à l'égard de la culture locale et du territoire. Nous présenterons ici les résultats de notre enquête et nous verrons les points qui confirment cette hypothèse et ceux qui s'en écartent. Nous aborderons les trois types de projets et nous présenterons leurs liens avec plusieurs points : le sentiment d'appartenance, l'approche de la communauté locale, en intégrant pour chacun le discours sur les établissements scolaires, les représentations spatiales, mais aussi nous intègrerons certaines autres variables. Disons immédiatement que sur les trente jeunes interrogés, 18 étaient plutôt «en projet », 8 étaient plutôt «en projet médian » et 4 étaient plutôt « en anti-projet ».

\section{Les collégiens « en projet »}

23 Au regard de notre échelle d'attitude, les 18 jeunes "en projet » présentent une forte autonomie dans leur choix scolaire. Ils sont satisfaits de leur établissement, de la scolarité et de leur orientation. Pour eux, la réussite passe surtout par l'école, et ils ont le sentiment d'apprendre. Ils désirent rester à l'école, continuer des études, ce qui est un atout pour trouver du travail. Toutefois, si ces 18 jeunes sont fortement participatifs à l'égard de leur scolarité, les histoires résidentielles et les images spatiales apparaissent divergentes et se scindent en deux tendances.

24 a) Dans une première catégorie, nous avons 11 jeunes. Le tableau 1 présente quelquesunes de leurs caractéristiques. Deux éléments sont intéressants, le temps d'installation dans la ville et l'orientation en classe de seconde. On constate que pour la plupart de ces élèves, le fait d'être installé depuis leur naissance dans cette ville et l'orientation vers Gide sont en relation avec la dimension projet. Mais ces éléments sont-ils vécus comme significatifs? 


\begin{tabular}{|c|c|c|c|c|c|c|}
\hline$N^{\circ}$ & SEXE & $\hat{\mathrm{A} G E}$ & P.C.S. DU PËRE & TEMPS D'INSTAL. & LIEU D'HABITAT & ORENTATION \\
\hline 1 & F & 16 & Entrep. Bât. & toujours & Centre & Gide \\
\hline 2 & M & 15 & Entrep. Bât. & toujours & Extérieur & Gide \\
\hline 3 & F & 17 & Plâtrier & toujours & H.L.M. & Gide \\
\hline 4 & F & 14 & Notaire & toujours & Centre & Gide \\
\hline 5 & F & 14 & Plombier & 1996 & H.L.M. & Gide \\
\hline 6 & $\mathrm{M}$ & 16 & Taille pierre & toujours & $1^{\text {er }}$ Cercle & Gide \\
\hline 7 & $F$ & 15 & Architecte & 1995 & $1^{\text {er }}$ Cercle & Gide \\
\hline 8 & $\mathrm{~F}$ & 15 & Maçon & toujours & $1^{\text {er }}$ Cercle & Gide \\
\hline 9 & $F$ & 15 & Commerçant & toujours & Extérieur & Gide \\
\hline 10 & $\mathrm{M}$ & 13 & Médecin & 2001 & Centre & Gide \\
\hline 11 & F & 14 & Agent immo. & toujours & Centre & Gide \\
\hline
\end{tabular}

Caractéristiques des jeunes en projet ( $\left({ }^{\mathrm{re}}\right.$ catégorie)

Characteristics of the youth involved in a project (first category)

Uzès est considérée comme la plus belle ville et ces jeunes mettent en exergue les éléments de son patrimoine: "J'aime bien le style des bâtiments, c'est l'esprit d'ici». Ils se sentent liés aux pierres et à la mémoire des lieux : "J'aime bien passer dans les petites rues, c'est des raccourcis que les gens d'ailleurs ne connaissent pas ». Au-delà de ce microcosme, ils vivent leur cité par la grandeur qu'elle déploie. «Mon père m'a expliqué beaucoup de choses sur le Duché (...) Le marché est le plus grand de France». Tous ont un fort sentiment d'appartenance à cette cité, car non seulement ils sont (pour beaucoup) là depuis leur naissance, mais ils portent en eux une part de cette expérience urbaine: «je me sens appartenir à Uzès, ça fait longtemps que je suis là ». "Je me sens appartenir à cette ville, d'ailleurs j'ai connu le Comte et la Comtesse, j'ai joué dans la cour du Duché». Dans cette connivence entre les jeunes et leur ville, le lycée $C$. Gide devient une des références. D’ailleurs, on a l'impression qu'il n'existe que cet établissement scolaire. Il est perçu de manière fortement positive. C'est un bon lycée, mis en valeur par son atmosphère paisible et sa réputation: "Gide, c'est calme ». "On m'a dit que c'est un bon lycée, et pour la seconde il vaut mieux rester ici, il y a de bons profs et plus de réussite ». Ce qui le distingue, c'est qu'il est protégé de l'envahissement de la sphère professionnelle : "On m'a dit que les classes étaient protégées. J'imagine que ça doit être bien ». "Gide, comme lycée c'est le mieux au moins il n'y a pas de professionnel ». Mais le plus significatif est que cet établissement leur permet de rester sur place : «Je veux aller à Gide pour rester ici». Ainsi cette orientation les fait entrer dans le temps du rêve : «je voudrais reprendre un cabinet d'huissier à Uzès ». "Je veux faire architecte et avoir mon cabinet sur Uzès ».

Comme tout sentiment d'appartenance puissant, ces jeunes vont alors construire une distance avec ceux qui ne sont pas d'ici, ceux qui ne font pas partie de cette communauté. C'est le cas ponctuellement des touristes, mais au-delà ce sont les étrangers, "les autres ", qui portent ce stigmate. Dans cette activité de préservation, on peut comprendre que le lycée professionnel soit peu connu : "Je crois qu'il y a un autre lycée en face de la mairie ». De toute manière, il est appelé le L.E.P., l'autre, ou le professionnel: «le L.E.P., je connais personne qui y est allé». "Ah oui ! il y a l'autre ». Son image est celle d'un établissement de parias qui a mauvaise réputation. C'est le monde de la racaille et des drogués : " Guynemer, on dit que les profs sont bizarres, il y a une mauvaise ambiance, et c'est une réputation de 
drogués ». Pour sa part, le Grand Mas est pratiquement absent. Pour la seule élève qui en parlera, c'est : «celui qui est à l'extérieur ».

Lorsqu'on porte un regard sur les cartes mentales de ces jeunes, on constate que les images de la ville (schéma 1) sont plutôt de type circulaire. Les voies de communications sont réduites et convergentes vers l'intérieur. La frontière est le boulevard qui englobe la cité. Elle permet de délimiter ce qui est de l'ordre de l'intérieur et ce qui est de l'ordre de l'extérieur. Si la plupart d'entre eux font apparaître un ou plusieurs établissements scolaires, le lycée Gide est le plus souvent représenté. Enfin, les éléments du patrimoine ont une place importante. La ville enlace les individus, elle leur permet de se réfugier à l'intérieur d'un lieu qui n'est pas accessible à tous. Elle est réservée.

Ces jeunes se présentent dès lors comme les élus de la communauté locale. Leur motivation n'est pas seulement d'aller à Gide pour continuer des études, mais c'est surtout la possibilité d'entretenir ce site d'appartenance. Ils désirent s'attacher à cette forme communautaire : «Partir serait un déchirement ».

Figure 1

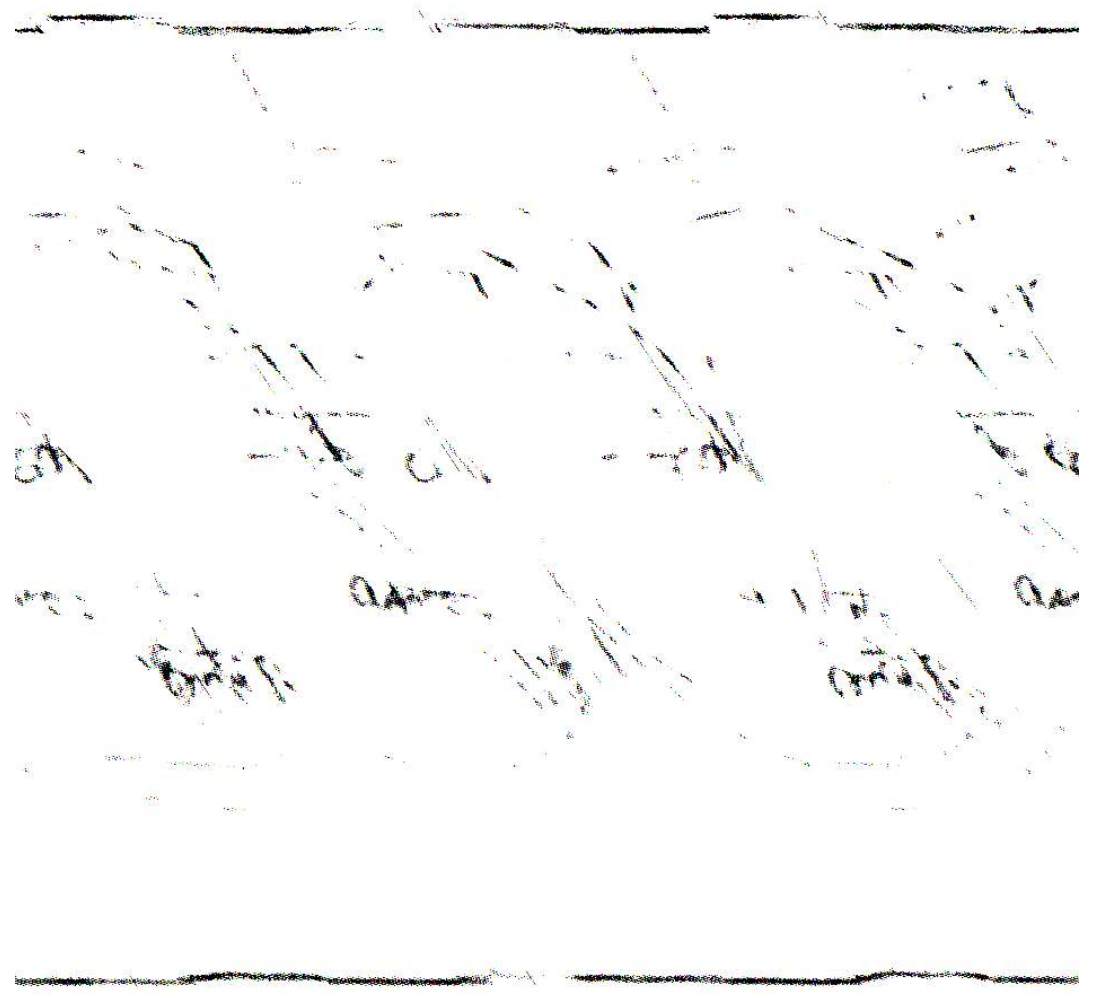

Ville circulaire

Circular Town

Ils reconnaissent la communauté et inversement, sont reconnus par elle. Ils sont, d'une part, soutenus par la lignée familiale comme les parents, les sœurs, les frères, les grands parents, les cousins, les tantes, les oncles, mais aussi par des réseaux d'amis des parents, des réseaux de relations des parents (communauté imaginaire, clients, patients). Nous avons alors affaire à un ensemble de parentés fictives (Wirth, 1938). Ils intériorisent le discours professoral du collège, d'autre part, quand ils disent: "On nous parle peu de l'orientation, mais c'est surtout pour faire une seconde générale à Gide ». Chez ces jeunes, il ne devrait exister aucun problème. Or, cette situation semble sous tendue par une forme de 
peur de se retrouver à l'extérieur de la ville, de leur ville : «Partir ça m'effraye ». Certaines études pointaient ce fait chez des jeunes marginalisés ou disqualifiés qui refusaient de quitter leur lieu de vie, car ils pouvaient garder localement nombres de soutiens. Or, ils souscrivent à la même logique : "On nous fait peur et on nous dit : si vous travaillez pas vous ne pourrez pas aller à Gide». N'entrent-ils pas dans une forme de repli communautaire qui peut dévoiler quelques problèmes d'intégration sociale?

b) Dans la seconde catégorie, comme le précise le tableau 2, la différence est sensible au regard du temps d'installation dans la ville qui est plus récent que précédemment, mais aussi compte tenu de l'orientation qui se fera majoritairement dans un lycée classique à l'extérieur de la ville. Ces éléments se retrouvent-ils dans les histoires biographiques des jeunes?

31 Ces jeunes définissent cette ville comme un monde de la réduction et de l'ennui : «Uzès, c'est un peu petit ». L'ambiance y est pesante : « Ici il n'y a pas grand chose à faire. On a vite fait le tour. L'hiver, c'est mort et c'est déprimant ». L'histoire, la mémoire ou l'art ne les concernent pas. "L'histoire d'Uzès ne m'intéresse pas, et partir ça ne me dérange pas ». «J'aime pas les vieilles choses ». Ces élèves ne se sentent ni appartenir à ces lieux, ni à une quelconque culture locale. Parallèlement, les lycées Gide et Guynemer ne sont que deux établissements scolaires sans particularités évidentes. Pour certains, ce ne sont pas de bons établissements : «Leurs réputations ne sont pas bonnes ». Pour d'autres, ils sont peu connus : "Gide et Guynemer je les connais pas trop ». "À Guynemer je ne sais pas ce qu'ils font et à Gide il n'y a pas toutes les sections ». Pour sa part le Grand Mas n'est que légèrement présent.

Tableau 2/Table 2

\begin{tabular}{c|c|c|c|c|c|c}
\hline No $^{\circ}$ & SEXE & $\hat{A} G E$ & P.C.S. DU PÈRE & TEMPS D'INSTAL. & LIEU D'HABITAT & ORIENTATION \\
\hline 12 & $\mathrm{M}$ & 14 & Cadre sup. & 2001 & $1^{\text {er Cercle }}$ & L.C. Ext. \\
\hline 13 & $\mathrm{M}$ & 15 & Cadre. Comm. & 2002 & $1^{\text {er Cercle }}$ & L.C. Ext. \\
\hline 14 & $\mathrm{~F}$ & 14 & Médecin & 1999 & Centre & L.C. Ext. \\
\hline 15 & $\mathrm{M}$ & 15 & Enseignant & 2000 & $1^{\text {er }}$ Cercle & L.C. Ext. \\
\hline 16 & $\mathrm{M}$ & 14 & Entrep. Bât. & 1998 & Extérieur & L.C. Ext. \\
\hline 17 & $\mathrm{~F}$ & 15 & Retraité & 1996 & H.L.M. & L.T. Ext. \\
\hline 18 & $\mathrm{M}$ & 16 & Retraité & 1995 & H.L.M. & L.P. Ext. \\
\hline
\end{tabular}

Caractéristiques des jeunes en projet ( $2^{\mathrm{e}}$ CATÉgoRIE)

Characteristics of the youth involved in a project (second category)

Les critiques se concentrent surtout sur la communauté locale qui est formée par les "gens d'ici». Ces jeunes souffrent des commérages, des rumeurs malsaines, et c'est un univers trop petit pour eux : "Les seuls gens normaux sont ceux qui ne sont pas d'ici». Dans cette perspective, Gide est simplement bon pour : «Ceux qui veulent rester ». Que ces jeunes aillent vers des filières technologiques ou classiques, l'ensemble a choisi ce départ : "Je préfere partir, ça me fera connaître d'autres personnes». Leur idée principale est celle de partir et ils rêvent d'un ailleurs: "je veux pas rester trop longtemps ici». "Je veux faire médecin et installer mon cabinet dans une grande ville». «Moi, je veux faire du commerce dans une ville importante ».

À la différence des autres jeunes en projet, la forme globale des cartes mentales est ici de type circulation (schéma 2). Les rues et les boulevards qui sont représentés ne sont que des axes de mobilité. S'il existe une frontière délimitée par la place centrale, les voies de 
communication centrifuges permettent de s'extirper de cet espace. Les éléments du patrimoine et les établissements scolaires sont peu représentés (seulement 2 dessinent un lycée). La ville ne devient qu'un lieu de passage, où tout enracinement est impossible et non souhaité.

Ces élèves se présentent comme des rebelles à l'égard de cette communauté locale. Ils la vivent de l'extérieur car ils ne sont pas originaires de cette localité : «Depuis que je suis ici je me suis toujours senti mis à l'écart». Ce ne sont pas des enracinés, et leur réseau local est limité : "Pour s'intégrer c'est particulier, il y a une fermeture comme un clan ou un noyau». D'ailleurs leurs parents semblent avoir aussi certaines difficultés d'intégration. : "Ma mère ne supporte plus d'être ici ». "Mes parents ne se sont jamais senti admis dans ce milieu ». Pour ces jeunes, les informations doivent être recherchées ailleurs que dans cette ville ou que dans leur collège puisque : "Quand le prof principal prend un exemple c'est toujours Gide qui est pris, alors qu'il y a d'autres lycées". Ainsi, en l'absence de soutien, d'aide locale ou d'information, ces jeunes envisagent tous de partir. Mais en décidant d'aller dans d'autres localités, ne veulent-ils pas se libérer de cette culture locale? Le choix d'orientation ne déborde-t-il pas la seule logique scolaire? Si pour certains, la filière scolaire n'existe pas dans cette localité, pour la majorité d'entre eux, leur inscription future se fera dans un établissement d'enseignement général. En cela, ils expriment plutôt un désir de distanciation à l'égard du lieu de résidence.

Figure 2

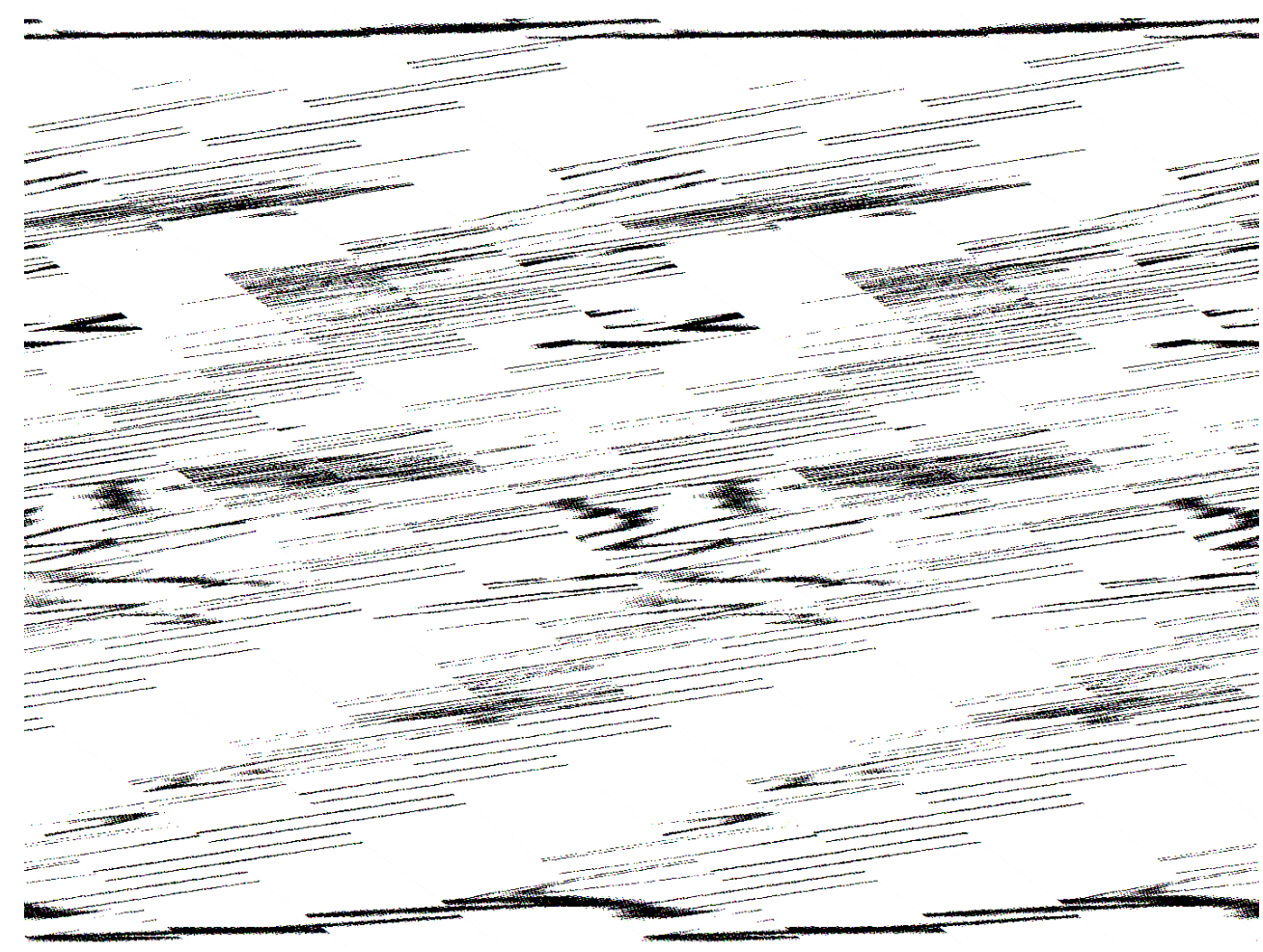

Ville circulation

Circulation Town 


\section{Les collégiens « en projet médian »}

35

Les jeunes "en projet médian» ont un sentiment ambigu à l'égard de leur autonomie scolaire, ils oscillent entre suivisme et liberté de choix. Les idées à l'égard de leurs établissements, de la scolarité en général, ou de l'apprentissage à l'école varient nettement, de la satisfaction au dépit. Toutefois, qu'ils soient satisfaits ou pas, ils désirent continuer sans vraiment s'occuper de ce qui se passe. La variabilité de ces attitudes se retrouve dans la représentation qu'ils ont de leur avenir, mais aussi dans la vision qu'ils ont du rapport entre école/emploi et utilité de l'école.

Dans ce cas, les caractéristiques (tableau 3) similaires viennent de la P.C.S. des pères (employés), de leur temps d'installation (né sur place), de leur lieu d'habitation ( $1^{\text {er }}$ cercle), de leur orientation en seconde (à l'extérieur). Ces éléments se retrouvent-ils dans les récits de vie?

Tableau 3/Table 3

\begin{tabular}{c|c|c|c|c|c|c}
\hline No $^{\circ}$ & SEXE & $\hat{\text { A GE }}$ & P.C.S. DU PĖRE & TEMPS D'INSTAL. & LEU D'HABITAT & ORIENTATION \\
\hline 19 & $\mathrm{~F}$ & 14 & Boulanger & toujours & $1^{\text {er }}$ Cercle & L.P. Ext. \\
\hline 20 & $\mathrm{~F}$ & 14 & Ferronnier & toujours & $1^{\text {er }}$ Cercle & L.T. Ext. \\
\hline 21 & $\mathrm{M}$ & 14 & Employé & toujours & $1^{\text {er }}$ Cercle & L.T. Ext. \\
\hline 22 & $\mathrm{~F}$ & 15 & Employé & toujours & $1^{\text {er Cercle }}$ & L.P. Ext. \\
\hline 23 & $\mathrm{~F}$ & 14 & Employé & toujours & $1^{\text {er }}$ Cercle & L.P. Ext. \\
\hline 24 & $\mathrm{M}$ & 15 & Employé & 1996 & Centre & L.T. Ext. \\
\hline 25 & $\mathrm{M}$ & 16 & Exp. Agric. & toujours & Extérieur & L.T. Ext. \\
\hline 26 & $\mathrm{~F}$ & 14 & Employé & toujours & $1^{\text {er Cercle }}$ & L.P. Ext. \\
\hline
\end{tabular}

Caractéristiques des jeunes en projet médian

Characteristics of the youth involved in a median-project

Lors des entretiens, ces jeunes mettent immédiatement en exergue l'histoire de leur ville. «J'ai des livres d'Uzès ». "Je connais l'histoire d'Uzès et j'ai un livre sur Anne de Crussol ». "Je vais à la médiathèque où j'ai trouvé des photos anciennes de ce qui se passait à Uzès ». "Je me suis intéressé à l'histoire d'Uzès, quand les Protestants se cachaient dans la crypte ». S'ils sont originaires des lieux, leurs parents aussi font partie de cet endroit. L'enracinement et le sentiment d'appartenance sont puissants: «Mes racines sont là. Le père de mon père était commerçant ici ». Pour ces élèves, Gide n'est ni un bon lycée, ni un vrai lycée, c'est « Le » lycée classique. C'est le monde des lettres et de la littérature, dominé par la culture du grec et du latin: «Gide, c'est le lycée. On y va surtout pour l'option grec ». "C'est fait pour ceux qui veulent faire du latin ». Pour sa part, si Guynemer reste toujours cet autre, il n'est pas dévalorisé. C'est plutôt le monde des arts et des artistes. "Le professionnel c'est plus le domaine des arts ». " Au professionnel, il y a surtout des métiers d'arts ». Le Grand Mas est par contre totalement absent.

Mais, au-delà de cette empreinte territoriale, ce petit microcosme semble se casser. La ville se brise en deux : «Ici il y a deux Uzès ». «Le Duché pour y habiter il faut avoir du sang noble, et je n'en ai pas ». "Ici, il y a ceux qui sont riches et puis les autres». Ces jeunes sont dépossédés de leur espace et certaines formes d'appropriation les transforment en exclus : « Moi je me sens exclue de la communauté des bourges, ceux qui 
sont propriétaires d'Uzès ». Ils basculent alors entre le regret et la critique. "C'est beau, mais il y en a qui volent la ville. Ça m'énerve un peu ». «Ici c'est ma vie, mais des fois je me sens plus chez moi ». On observe une dissonance identitaire, une forme de défense pathétique, où l'individu désire rester alors que ce local ne le lui permet pas : « La justice est mal faite. Pourquoi il faut que je parte alors que d'autres peuvent rester et ils sont pas d'ici ?». En effet, le problème est que pour accéder à un établissement de la localité, il faut, selon eux, soit avoir de très bons résultats scolaires, soit avoir la fibre d'un artiste : « Mes résultats sont trop faibles pour aller à Gide ». « Le lycée professionnel, c'est surtout pour les artistes ». Paradoxalement, ces jeunes ont choisi leurs filières scolaires et partent vers des filières technologiques ou professionnelles à l'extérieur, alors qu'il subsiste chez eux ce regret de ne pas pouvoir rester : «Ça m'embête de partir d'ici et pour moi ça a été un gros coup ".

Lorsqu'on observe les plans réalisés, la ville est dépeinte de manière " quasi circulaire " (schéma 3). Nous n'avions pas prévu ce type de plan, et lors de nos recherches antérieures, nous avions certainement interprété ces dessins comme s'il s'agissait de plans de type circulaire. En effet, à la différence des jeunes en projet de la première catégorie, s'il existe bien l'image du rond, les éléments représentés sont peu en liaison les uns avec les autres. On constate en fait qu'il y a des trous dans l'espace présenté. Les voies de communication se résument en partie à un segment de boulevard, ou à quelques rues et impasses. La frontière est constituée par le boulevard et édifie un mur qui bloque l'accès, dans le cas typique du collège aux lycées mais aussi aux monuments du patrimoine qui y sont fortement mis en évidence. Mais cette frontière ne relève-t-elle pas aussi la difficulté d'accéder au « centre »? La ville est segmentée et ne leur appartient plus. Elle s'effrite.

Figure 3

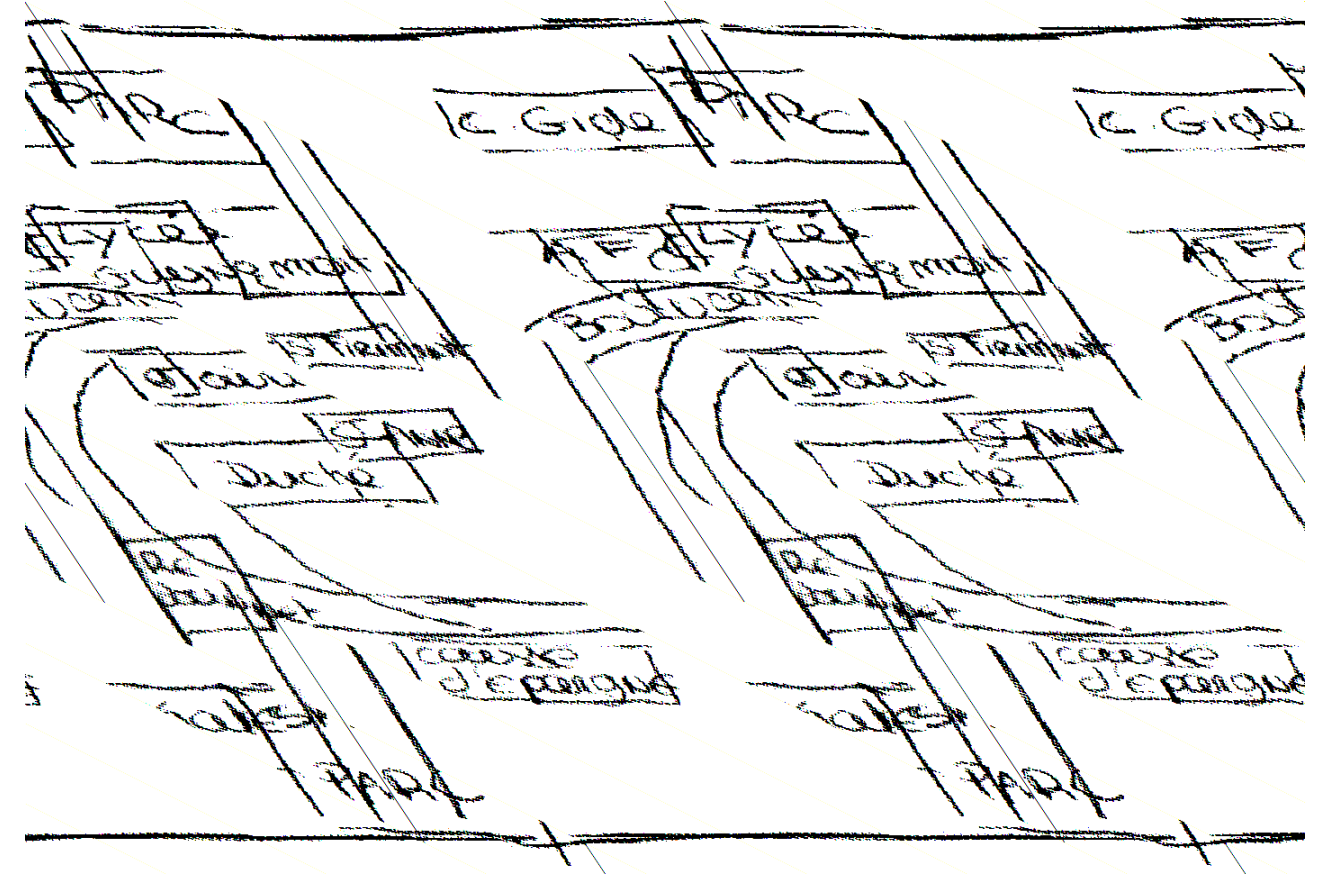

Ville quasi circulaire

Quasi-Circular Town

Ces jeunes pensent qu'ils ne font plus partie de la communauté territoriale, alors qu'ils sont pratiquement tous originaires de cette cité. S'ils ont un net sentiment 
d'appartenance, ils se sentent en même temps en proie au déracinement. Les informations qu'ils reçoivent sont éparses et seuls quelques relais locaux ou familiaux entrent en jeu. Là nous avons un "entraîneur de vélo", ici "une voisine », ici encore "une tante ». Situation d'autant plus frustrante que cette localité est fortement valorisée. On ne peut qu'observer un fort sentiment de frustration et d'injustice. L'idéalisation du territoire est importante au même titre que l'école locale : "Mon oncle était à Gide et il était content d'être là ». Or, ils ont l'impression que leur mobilité scolaire est imposée. Ces jeunes vivent alors leur situation comme fortement ambiguë. Tous admettent qu'ils auraient préféré rester, et tous sont amenés à quitter leur ville. La tension n'en paraît que plus forte. En cela, lors même qu'ils choisissent leur filière scolaire, ils ont tous le sentiment de subir cette orientation : "Les professeurs nous montrent qu'une voie (...) c'est choisir $S$ à Gide et si t'as pas les capacités ou si tu ne veux pas alors t'es incapable et t'as qu'à partir». Cette exclusion de l'école locale est alors vécue comme un exil forcé. Lorsqu'ils doivent partir, ils tentent pourtant de recouvrer une part de la communauté en évitant d'être interne ou en espérant un retour. L'espoir de revenir sur place se mêle à la critique de ceux qui prennent leur place.

\section{Les collégiens « en anti-projet »}

41 Les jeunes "en anti-projet " ont une faible autonomie dans leur choix scolaire et ont le sentiment d'être sous pression constante. Ils ne sont pas satisfaits de l'établissement, de la scolarité, et n'attribuent que peu de sens à leurs études. La réussite ne passe pas par l'école, mais être scolarisé leur permet de faire quelque chose. Ils envisagent peu leur avenir scolaire, car cette situation n'est que peu en relation avec leur travail futur.

Dans ce quatrième cas, on peut constater (tableau 4) que les pères sont tous ouvriers, qu'ils vivent dans l'aire des H.L.M., et qu'ils vont à l'issue de leur troisième vers des lycées professionnels à l'extérieur de la ville. Or, ces dimensions sont-elles apparentes lorsqu'on tient compte de leurs histoires résidentielles?

Tableau 4/Table 4

\begin{tabular}{c|c|c|c|c|c|c}
\hline No $^{\circ}$ & SEXE & $\hat{\text { AGE }}$ & P.C.S. DU PĖRE & TEMPS D'INSTAL. & LIEU D'HABITAT & ORIENTATION \\
\hline 27 & $\mathrm{M}$ & 15 & Ouvrier Maçon & toujours & H.L.M. & L.P. Ext. \\
\hline 28 & $\mathrm{M}$ & 16 & Ouvrier Agric. & 1995 & H.L.M. & L.P. Ext. \\
\hline 29 & F & 15 & Ouvrier Agric. & toujours & H.L.M. & L.P. Ext. \\
\hline 30 & $\mathrm{M}$ & 17 & Ouvrier Agric. & toujours & H.L.M. & L.P. Ext. \\
\hline
\end{tabular}

Caractéristiques des jeunes en anti-projet

characteristics of the young «involved in a anti-project»

Ces élèves vivent cette ville comme un espace étroit : «J'ai toujours vécu ici mais il n'y a rien à faire. Je reste chez moi et je dors ". "Uzès c'est trop petit et il n'y a rien à faire ». «C'est petit et c'est un territoire paumé et pourri». Ils ont un faible sentiment d'appartenance à cet espace territorial. Paradoxalement, le seul moment de l'année où ils se sentent bien c'est lorsqu'il y a des touristes. Ainsi, ils peuvent se mêler à ceux-ci et faire comme s'ils venaient d'ailleurs : "Je suis contente quand il y a des touristes ». "Les touristes c'est bien. Ils parlent différemment, ils ont d'autres langues et d'autres manies ». Le discours sur les établissements scolaires est le pendant des allusions sur leur ville. Pour ces élèves, on a la vive impression que les lycées disparaissent de leur champ 
de référence quotidien. «On parle surtout pas des lycées». Ici, ce n'est plus l'image des lycées qui est mise en perspective mais leur vécu de collégien: "Au collège de toute façon, on se sent pas libre, c'est trop laid et les profs sont sévères ».

Dans ce contexte, tout espoir semble vaincu car : « En fait, il y a beaucoup pour les uns et peu pour les autres ». Ils ne peuvent accéder à la collectivité locale qui ne leur offre de toute manière aucune place, car non seulement ils n'appartiennent pas à la mémoire des lieux, mais sont aussi étrangers à l'espace central. Sur ces quatre jeunes, trois sont issus de l'immigration, et tous vivent dans des habitations bordant la vieille ville : "Je suis pas allé à l'office du tourisme, mais je crois qu'ils m'interdiraient d'entrer ». Les informations semblent rester aux portes de la vieille ville. Ainsi, ces jeunes admettent que les informations qu'ils possèdent sur les lycées sont faibles : «Les lycées, je ne sais pas ce qu'il y a dedans ». "je crois qu'à Gide il y a une seconde et à Guynemer je connais pas les filières ». Mais ils savent aussi que de toute façon ce n'est pas pour eux : «Tout le monde peut pas y aller ». «Il y a les bouffons, les riches et les autres ils sont mis à l'écart ». Ainsi, méconnaissance et mise à l'écart sont vécues comme des formes de mépris. «L'école ça m'énerve, on le dit tous. Les profs, ils parlent trop et je comprends rien à ce qu'ils disent. C'est un peu comme s'ils me tenaient à distance ».

Quand on analyse les dessins de ces élèves, la forme d'ensemble recouvre l'image de l'éclatement (schéma 4). Les voies de communication sont inexistantes et il n'y a pas de lien entre les blocs dépeints. La place centrale fait office d'espace fermé et inaccessible. Il n'y a pas d'entrée et la frontière est nette avec les autres aires. Les points de repère ne sont ni les bâtiments du patrimoine, ni les lycées, ni les collèges, mais les lieux d'habitation et les espaces de jeux. La ville n'est plus qu'un ensemble de petits territoires sans liens les uns aux autres, sans véritable cohésion. Elle est déstructurée.

Figure 4

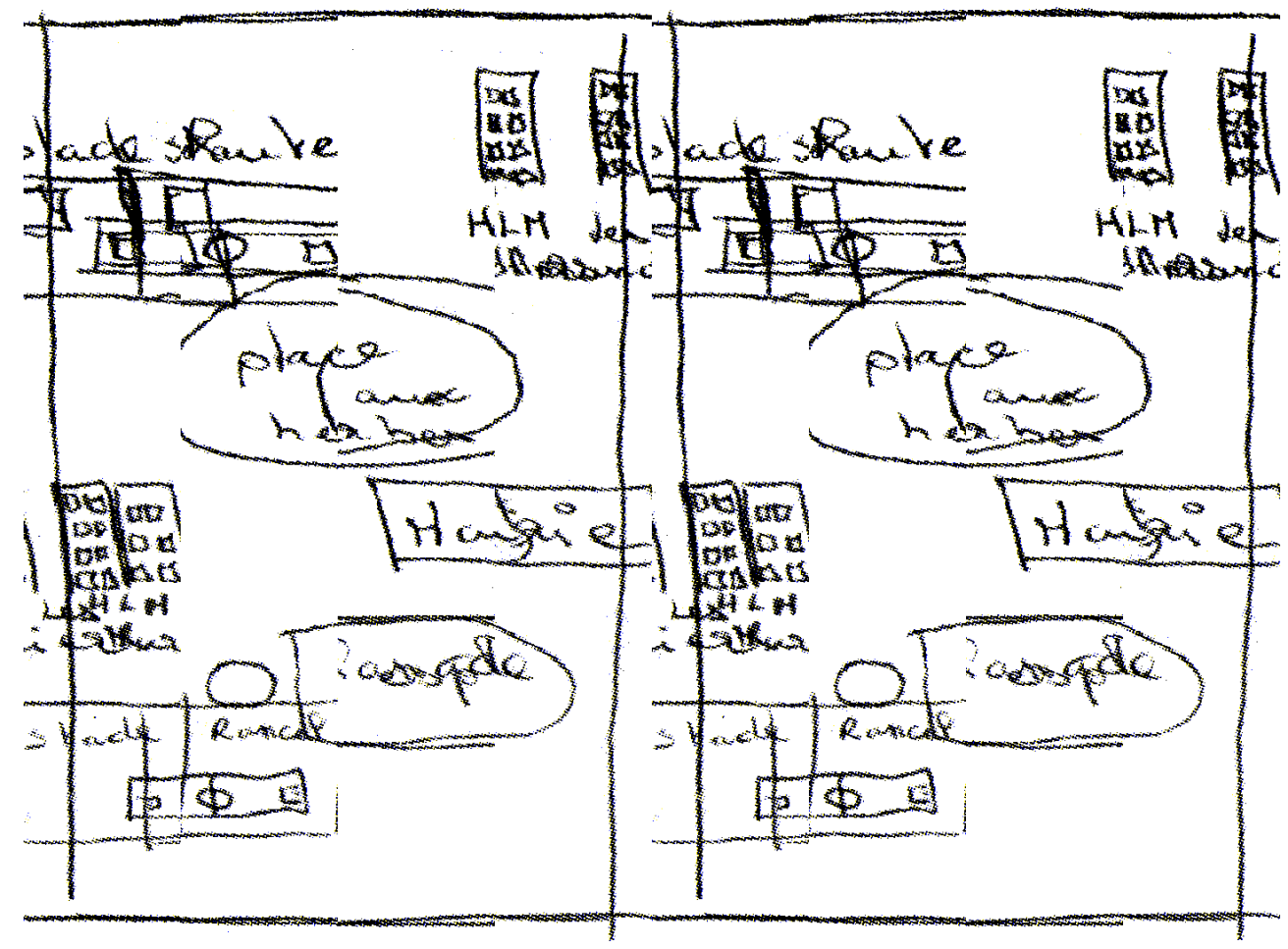

Ville éclatée

Scattered town 
46

jeunes ont la nette impression qu'ils ne font pas partie, et qu'ils n'ont jamais fait partie de la culture locale. Ils sont isolés dans leur espace en limite de la " cité ancienne ", ils sont aussi socialement mis à l'écart et restent des étrangers. L'esprit du local est vécu totalement de l'extérieur : «Personne ne me donne d'information ». De toute façon, comme ils le disent: "il n'y a pas de place pour nous à Gide». L'exclusion sociale se double d'une exclusion scolaire : "Quand on n'a pas de bonne note, on nous dit qu'on ira en L.P. " "On me dit souvent que même au L.E.P. d'ici, ils voudrons pas de moi». Ces élèves se sentent certes méprisés mais surtout exclus de cette communauté locale. Ils se vivent comme des maudits que l'école ne peut racheter. Ils perçoivent leur situation comme s'ils étaient des parias. Pour eux, ils n'ont de place ni dans la cité ni dans l'école. Dans cette exclusion simultanée, ils entrent dans une logique qui se compose de honte et de culpabilité. Ils sont les vaincus et entretiennent à l'égard de leur ville et de la scolarité de multiples formes d'inimitiés. Ils entrent dans des formes de compensation (Gans, 1968) et de repli sur soi, mais aussi développent des animosités et rejettent les autres.

\section{Conclusion}

À partir des divers résultats de cette enquête, que pouvons-nous dire du lien entre les attitudes scolaires, interprétées en termes de projet, et le contexte spatial ? Que pouvonsnous dire des rapports entre les jeunes, leurs histoires résidentielles, l'école et la culture locale?

On peut admettre que le sentiment d'appartenance, le degré d'enracinement au territoire et à la culture locale, mais aussi la manière de représenter et de vivre son espace quotidien, influencent les attitudes juvéniles. Cela étant, il faut relever, dans un premier temps, une exception en ce qui concerne la seconde catégorie de jeunes en projet. Isolons tout d'abord ces derniers et abordons les autres.

On constate que plus l'attachement au local et le sentiment d'appartenir à la communauté locale sont forts, plus le jeune aura tendance à être participatif à l'égard de sa scolarité et plus il sera "en projet" avec l'école. Ce point confirme les analyses canadiennes qui montrent que plus les jeunes se sentent attachés à leur localité et plus ils désirent y rester, parce que c'est un lieu d'épanouissement et une source d'emploi futur (Camiré, Roy \& Ouellet, 1994, p. 32). Lorsque cet attachement ou ce sentiment d'appartenance vient à se gripper, comme dans le cas où il existe une rupture entre l'enracinement territorial et l'enracinement généalogique (Centlivres, 1986), le jeune vient à perdre pied. Ses conduites oscillent et il entre " en projet médian ». Quand l'attachement et le sentiment d'appartenance aux lieux deviennent faibles, les attitudes des élèves sont proches du retrait et de ce que nous avons nommé : «anti-projet ». Dans ce contexte, il y a semble-t-il un glissement progressif dans lequel les collégiens passent d'une capacité à pouvoir s'orienter dans l'espace local et à l'école à une désorientation dans les deux mondes. L'appel aux cartes mentales confirme, en bien des points, ces éléments où l'image d'une réalité donnée peut varier lorsqu'on change les circonstances de vision (Lynch, op. cit, p. 56). En effet, entre la ville circulaire, la ville quasi circulaire et la ville éclatée, on observe la même modification. D'un espace ramassé et englobant, on glisse petit à petit vers un espace segmenté qui s'effrite, puis vers un espace totalement diffus. Le type de rapport qu'entretient alors l'individu avec son environnement influence directement les conduites scolaires et le désir de participer, de s'engager (Zaffran, op. cit., p. 43), ou bien

L'orientation scolaire et professionnelle, 35/1 | 2006 
de se rétracter. Chacun sélectionne des éléments de son environnement immédiat pour considérer ou reconsidérer la situation dans laquelle il se trouve.

Il subsiste un cas particulier qui est celui des jeunes «en projet » de seconde catégorie. Ils ont pour leur part un faible sentiment d'appartenance et ne se reconnaissent pas dans cette culture locale. Apparemment l'histoire résidentielle différente de ces jeunes affecte les projets d'une manière divergente. Le sens de la participation scolaire n'est, de ce fait, pas le même. Si pour les enracinés l'affectation dans le lycée local leur permettait de rester attachés à la culture locale, et l'effet local était attractif et les sécurisait (Moquay, 1997, p. 247), pour les non enracinés le projet ne possède pas la même signification. Pour ceux qui ne se sentent pas attachés à la localité, par absence de repères généalogiques ou territoriaux, la participation scolaire leur permet de partir de cette collectivité locale. Ici, l'effet du local joue donc un rôle répulsif. Or, s'ils ne sont pas originaires des lieux, à la différence de bien des autres jeunes de notre population, s'ils sont étrangers au local et ont déjà migré, ils possèdent surtout de nettes ressources parentales (P.C.S. des pères), à la différence des jeunes en anti-projet. Cette dimension leur permettrait d'éviter de glisser vers le retrait. Les cartes mentales mettent d'ailleurs en évidence ce rapport à la ville qui n'est perçu que comme un lieu de passage. On pourrait aussi proposer qu'ils ont a priori intégré et intériorisé la mobilité. L'attachement et l'enracinement ne seraient plus alors des ressources, mais deviendraient des contraintes.

51 Toutefois et malgré ces différences, on remarque que ces histoires de vie ne naissent pas de rien (Guillaume, 1998). Elles se réfèrent toutes, certes point sur lequel nous attachons notre démarche, au contexte spatial. Quant aux attitudes scolaires, elles se construisent sur la base de ces vécus et non pas seulement dans l'enceinte de l'école (Zaffran, op. cit.). Les niveaux de projets scolaires se structurent dès lors dans un espace temporel dont l'école n'est qu'une des parties. Ainsi, pour certains jeunes "en projet", l'école, le territoire et la culture locale sont en étroite osmose. L'activité de ces élèves se centre sur cette volonté de rester sur place, non pas dans une pure logique scolaire, mais surtout pour garder une place au sein de la communauté locale. Pour les autres collégiens «en projet ", les deux mondes que sont l'école et la ville sont tous deux des lieux de passage et de mobilité. L'action des élèves procède alors du détachement local et de l'engagement dans d'autres lieux. L'activité du projet permet d'envisager de partir avec de nouvelles ressources. Pour les jeunes "en projet médian", l'école et l'espace urbain sont vécus comme des entités sélectives et ségrégatives. La logique de l'action de ces élèves oscille pourtant entre le regret de partir et l'espoir du retour. Enfin, pour les jeunes «en antiprojet", l'espace de l'école comme celui de la ville ne deviennent que des lieux d'exclusion. L'activité des jeunes dans cette situation se centre ici sur la fuite, et le rejet de toutes formes de prise en charge.

Pour arriver à saisir et à expliquer les diverses facettes des attitudes scolaires de nombreux facteurs peuvent être mis en évidence. Certaines recherches mettent l'accent sur le sexe, sur l'âge, sur la nationalité, sur la profession ou le niveau scolaire des parents. D'autres pointent les effets des établissements, des classes ou des maitres. Notre approche loin d'infirmer ces études proposait de porter son regard en direction du contexte local. Toutefois, lorsqu'on fait référence à cet aspect territorial, le nombre et la répartition des offres restent souvent les facteurs privilégiés. Or, comme on l'a vu, la manière d'aborder et de comprendre la culture locale joue pour beaucoup dans la construction des conduites scolaires des jeunes. Si les diverses expériences sont situées et localisées, si ces jeunes 
vivent dans un espace particulier, le lieu semble les habiter ou les tenir à distance. Si certains collégiens doivent réaliser un véritable travail d'acculturation pour apprendre de nouveaux codes culturels et intégrer de nouvelles pratiques scolaires légitimes quand ils entrent au lycée (Beaud, 2002), les élèves de notre population sont obligés aussi de faire face à la culture des lieux, à ses valeurs et à ses histoires.

La notion de territoire met alors l'accent sur les façons dont les hommes s'approprient l'espace (Henriot-van Zanten, 1990), on pourrait ajouter aussi les manières dont ils s'approprient l'école. La culture des lieux est un des aspect de cette appropriation. Cette culture sous-tend un ensemble de valeurs, de codes, de présupposés ou de savoirs ordinaires qui n'est pas accessible à tous. Pour s'orienter sur un territoire, et donc dans cette culture particulière, il faut disposer de clefs (informations, connaissances, réseaux, coutumes, savoir-faire, dimension affective) et, sans elles, on ne peut s'identifier à cet espace. En l'absence d'identification, ou de perte d'identification, tout enracinement parait difficile, toute orientation semble perturbée. La culture des lieux construit de la ségrégation, de la segmentation, de la hiérarchisation. Cette construction et reconstruction est réalisée par les acteurs locaux, comme les médiateurs de la mémoire, une certaine élite locale, voire la presse. Ils contrôlent l'espace, le territoire. L'école ne devient-elle pas le relais de la sélection, de la ségrégation, ce qui entraîne bien des conséquences (Felouzis, 2003) ? Si pour les jeunes en projet de la seconde catégorie, on pourrait voir des stratégies d'évitement (Broccolichi, 1998), ne pourrait-on pas aussi repérer des stratégies de renforcement qui évincent certains jeunes et favorisent des transmissions d'une culture locale sélective ? L'école se plierait alors aux exigences d'une culture particulière qui favoriserait la ségrégation (Pinçon \& Pinçon-Charlot, 1989) et l'exil de certaines populations. On ne tiendrait pas compte de l'intérêt des élèves (Berthelot, 1993; Dumora, 2001), et les professeurs pourraient maintenir un monde nostalgique d'un âge d'or passé (Dubet, 2002, p. 137).

Mais cette culture locale est aussi entretenue par les jeunes eux-mêmes qui s'en réapproprient la dynamique. Pensons aux cas des établissements scolaires, de leurs places et de leurs images. Le lycée Charles Gide, cité par tous les élèves, semble être le noyau central de l'offre scolaire. Sa représentation est reliée à cette tradition locale qui fait de lui ce lieu particulier où: "On y étudie le latin et le grec». Pour sa part, le lycée professionnel Guynemer, même ayant pris la dénomination de lycée des métiers d'Art, reste « l'autre », « le professionnel », « le L.E.P. » et recouvre une représentation ambiguë faite d'artiste et de racaille. Le Grand Mas n'est cité que par peu d'élèves. Il est absent de l'imaginaire local. Les jeunes reconstruisent des cloisonnements et des hiérarchisations entre les établissements, les filières et les classes. Si l'école est dans la cité inversement la cité paraît s'installer dans les parcours scolaires. Ainsi, l'école n'est pas vécue comme une simple entité en soi et un lieu isolé. Elle est en interférence avec la dynamique spatiale dans laquelle elle se trouve. Les deux font corps, se mêlent, s'entremêlent, se rapprochent et s'écartent. L'imaginaire scolaire passe par cette forte hiérarchisation locale des établissements et des filières. Ainsi, les jeunes semblent intégrer et cette réduction des offres et ces formes de hiérarchisation véhiculées.

Mais cette crispation autour d'un territoire et d'une culture engendre corrélativement des problèmes importants et un cercle vicieux. Qu'ils choissent ou non leur orientation, qu'ils aient le sentiment de choisir ou non leurs filières, dans tous les cas, nous observons des formes de peur, d'angoisse, de rancœurs, ou d'agacement. Les conduites sont pour le moins pessimistes et les constructions identitaires sont défensives. La crainte et la 
frustration s'immiscent chez ces jeunes. On pourrait admettre qu'ils sont perméables à la conjoncture et qu'ils éprouvent une forte "crise identitaire». Une des conséquences est le net repli identitaire qui semble expliquer le besoin des jeunes de se définir à travers un espace matériel, qui donnerait du sens à leur vie quotidienne (Belot, 2005, p. 202). En effet, comme le montrent les comparaisons européennes, une forte proportion de jeunes affirme un net sentiment d'appartenance au niveau local (Ibid., p. 179). Or, si le local donne un cadre social et des références pratiques (Keating, 1997; Poche, 1996), il n'en demeure pas moins que les jeunes n'ont pas tous la même place au sein de cet environnement. Il faut qu'ils puissent accéder à des codes privés que seuls certains comprennent (Schütz, 1944, p. 29). Un des problèmes est que par l'intermédiaire de cette dynamique locale, les jeunes reconstruisent des rapports sociaux faits d'opposition entre " nous » et « eux ", entre ceux qui sont " d'ici » et ceux qui viennent "d'ailleurs », entre « moi » et les « autres». La cité (en partie par le biais de l'école) renforce le patriotisme local (Brown, 2002) et alimente par là l'image de l'altérité. Elle multiplie les rancœurs et provoque parfois de la haine. Elle stigmatise, méprise et exclut. De son côté, l'école est loin d'être un lieu de discussion, puisqu'elle renforce cet esprit particulier. Elle s'éloigne des seuls apprentissages, des seuls savoirs, des seules connaissances. Elle devient pour les acteurs locaux un enjeu territorial, et s'inscrit pour certains dans la défense du territoire, de leur territoire. Comment parler alors de citoyenneté à des jeunes qui en sont exclus, où se sentent exclus? Comment parler de justice à l'école alors que certains vivent son opposé au quotidien?

On a tendance à parler des cités et plus particulièrement des cités de banlieues avec leurs cortèges de problèmes. Ceux-ci entraîneraient des replis communautaires centrés, là sur l'ethnicité, là sur la religion. On omet la dynamique territoriale. Dans les petites villes, mais aussi dans des espaces plus étendus, on peut affirmer que les communautés territoriales jouent quelques partitions. Le local ré-émerge et reconstruit des sites d'appartenance qui affectent les attitudes scolaires des jeunes. On oublie alors trop souvent les déterminants de l'action liés au contexte spatial.

\section{BIBLIOGRAPHIE}

Bassoul, R., Bernard, P., \& Touraine, A. (1960). Retrait, conflit, participation, Trois types d'attitudes ouvrières au travail. Sociologie du travail, 2, 4, 314-329.

Beaud, S. (2002). 80 \% au bac... et après ? Les enfants de la démocratisation scolaire. Paris : La découverte.

Belot, C. (2005). Du local au mondial : Les espaces d'appartenances des jeunes européens. In Galland, O., \& Roudet, B. (Éds.), Les jeunes européens et leurs valeurs (pp. 177-203). Paris : La Découverte.

Berger, C., Fosse, N., \& Roques, J.-L. (1993). La santé et les jeunes dans le Gard Rhodanien. Rapport pour la D.D.A.S.S., Nîmes. 
Berger, C., \& Roques, J.-L. (2005). L'eau comme fait social : Transparence et opacité dans la gestion locale de l'eau. Paris : L'Harmattan.

Bernet, O. (2000). La représentation du problème « s'orienter » chez les lycéens de terminale. L'Orientation Scolaire et Professionnelle, 29, 3, 453-482.

Bertaux (2005). Le récit de vie. Paris : Armand Colin.

Berthelot, J.-M. (1993). École, orientation, société. Paris : P.U.F.

Biemar, S., Philippe, M.-C., \& Romainville, M. (2003). L'injonction au projet : paradoxale et infondée ? L'Orientation Scolaire et Professionnelle, 32, 1, 31-51.

Broccholichi, S. (1998). Inquiétudes parentales et sens des migrations d'élèves. Éducation et Formations, 101, 103-123.

Brown, P. (2002). La société et le sacré dans l'Antiquité tardive. Paris : Seuil.

Camiré, L., Roy, J., \& Ouellet, H. (1994). Les jeunes et l'exode dans le bas-Saint-Laurent : Étude de cas. Sainte Foy. Centre de recherche sur les services communautaires de l'Université de Laval.

Centlivres, P. (1986). L'identité régionale : « Langage et pratique ». In Centlivres, P. Les sciences sociales face à l'identité régionale : Cinq approches (pp. 77-126). Berne : Haupt.

Derouet, J.-L. (1988). Désaccords et arrangements dans les collèges. Revue française de pédagogie, 83, 5-22.

Dubet, F. (1973). Pour une définition des modes d'adaptation sociale des jeunes à travers la notion de projet. Revue française de sociologie, 14, 2, 221-241.

Dubet, F. (1991). Les lycéens. Paris : Seuil.

Dubet, F. (1994). Sociologie de l'expérience. Paris : Seuil.

Dubet, F. (2002). Le déclin de l'institution. Paris : Seuil.

Dubet, F. (2004). L'école des chances. Paris : Seuil.

Dubet, F., Cousin, O., \& Guillemet, J.-P. (1989). Mobilisation des établissements et performances scolaires. Revue française de sociologie, XXX, 235-256.

Dumora, B. (1990). Dynamique vocationnelle chez l'adolescent de collège. L'Orientation Scolaire et Professionnelle, 19, 2, 111-127.

Dumora, B. (1992). Le projet d'orientation du jeune : Réalité psychologique ou fiction pédagogique? In Boutinet, J.-P. (Éd.), Le projet (pp. 197-201). Paris : L'Harmattan.

Dumora, B. (2001). Les intentions d'orientation et leur argumentation. L'Orientation Scolaire et Professionnelle, 30, 2, 148-165.

Durkheim, E. (1893) (1998). De la division du travail. Paris : P.U.F.

Duru-Bellat, M., \& Mingat, A. (1988). Le déroulement de la scolarité en collège : Le contexte fait des différences. Revue française de sociologie. XXIX, 649-666.

Duru-Bellat, M., \& Van Zanten A. (2002). Sociologie de l'école. Paris : Armand Colin.

Duru-Bellat, M., Le Bastard-Landrier, S., Piquée, C., \& Suchaut, B. (2004). Tonalité sociale du contexte et expérience scolaire des élèves au lycée et à l'école primaire. Revue française de sociologie, 45, 3, 441-468.

Duru-Bellat, M. (2002). Les inégalités sociales à l'école. Paris : P.U.F.

Ehrenberg, A. (1995). L'Individu incertain. Paris : Calmann-Lévy. 
Felloneau, M.-L. (1994). Les étudiants et leurs territoires, la cartographie cognitive comme instrument de mesure de l'appropriation spatiale. Revue française de sociologie, 25, 2, 533-559.

Felouzis, G. (2003). La ségrégation ethnique au collège et ses conséquences. Revue française de sociologie, 3, 413-447.

Galland, O. (1991). Sociologie de la jeunesse. Paris : Armand Colin.

Gans, H.G. (1968). Urbanism and Suburbanism as Ways of Life. In Pahl, R. (Ed.), Readings in Urban Sociology (pp. 95-118). Pergamon Press.

Guichard, J. (1995). Psychopédagogie du projet d'avenir et normativité. In A.D.A.P.T./S.N.E.S., Les projets des jeunes (pp. 9-39).

Guillaume, J.-F. (1998). Histoires de jeunes. Paris : L'Harmattan.

Huteau, M. (1992). Les projets d'orientation des jeunes. In Boutinet, J.-P (Éd.), Le projet (pp. 33-47) Paris : L'Harmattan.

Halbwachs, M. (1925) (1994). Les cadres sociaux de la mémoire. Paris : Albin Michel.

Halbwachs, M. (1950) (1997). La Mémoire collective. Paris : Albin Michel.

Halbwachs, M. (1938) (1970). Morphologie sociale. Paris : Armand Colin.

Henriot-van Zanten, A. (1990). L'école et l'espace local. Lyon : P.U.L.

Javeau, C. (1992). L'enquête par questionnaire. Bruxelles : Éditions de l'Université de Bruxelles.

Kayser, B. (1990). La renaissance rurale. Paris : Armand Colin.

Keating, M. (1997). Les défis du nationalisme Moderne : Québec, Catalogne, Écosse. Montréal : Presses de l'Université de Montréal.

Lewis, O. (1963). Les enfants de Sanchez. Paris : Gallimard.

Lynch, K. (1960) (1998). L'image de la cité. Paris : Dunod.

Moquay, P. (1997). Le sentiment d'appartenance territoriale. In Gauthier, M. (Éd.). Pourquoi partir? Migration des jeunes d'hier et d'aujourd'hui (pp. 243-256). Sainte Foy : Presses de l'Université de Laval.

Namer, G. (1987). Mémoire et société. Paris : Méridiens Klincksieck.

Ostrowetsky, S. (1996). L'espace en tous sens. In Ostrowetsky S. (Éd.) : Sociologues en ville (pp. 255-276). Paris : L'Harmattan.

Park, R.-E. (1925) (2005). La ville. In Grafmeyer, Y., \& Joseph I. (Éds.), L'École de Chicago (pp. 83-130). Paris : Flammarion.

Peron, L. (1994). Brest, ville universitaire : Pratiques et représentations du campus de l'agglomération brestoise et de la région par les étudiants brestois. In Sechet, S. (Éd.), Université droit de cité (pp. 155-181). Rennes : P.U.R.

Pinçon, M., \& Pinçon-Charlot, M. (1989). Dans les beaux quartiers. Paris : Seuil.

Poche, B. (1996). L'espace fragmenté. Éléments pour une analyse sociologique de la territorialité. Paris : L'Harmattan.

Rochex, J.-Y. (1995). Le Sens de l'expérience scolaire. Paris : P.U.F.

Roncayolo, M. (1997). La ville et ses territoires. Paris : Gallimard. 
Roques, J.-L. (2003a). L'effet du local sur les projets de jeunes : Le cas de deux petites villes du Gard. L'Orientation Scolaire et Professionnelle, 32, 1, 3-30.

Roques, J.-L. (2003b). Modes d'expression de jeunes de petites villes. Sociétés, 81, 3, 105-115.

Roques, J.-L. (2004a). La petite ville et ses jeunes. Paris : L'Harmattan.

Roques, J.-L. (2004b). Une analyse des relations entre des jeunes, leurs projets et le contexte local. Revue Suisse de Sociologie, 30, 1, 99-122.

Schütz, A. (1944) (2003). L'étranger. Paris : Élia.

Stoetzel, J. (1963). La psychologie sociale. Paris : Flammarion.

Thomas, W.I., \& Znaniecki, F. (1919) (1998). Le paysan Polonais en Europe et en Amérique. Paris :

Nathan.

Van Zanten, A. (Éd.) (2000). L'école, l'état des savoirs. Paris : La Découverte et Syros.

Van Zanten, A., Payet, J.-P., \& Roulleau-Berger, L. (1994). L'école dans la ville: Accords et désaccords autour d'un projet politique. Paris : L'Harmattan.

Wirth, L. (1938). Le phénomène urbain comme mode de vie. In Grafmeyer, Y., \& Joseph I. (Éds.), L'École de Chicago, Paris : Flammarion, 255-281.

Zaffran, J. (2000). Les collégiens, l'école et le temps libre. Paris : Syros.

\section{NOTES}

1. Voir la conclusion de l'article de Duru-Bellat, Le Bastard-Landrier, Piquée \& Suchaut, (2004).

2. Une petite ville est définie à partir d'une population comprise entre 5000 et 20000 habitants.

3. Il est à noter qu'à la rentrée suivante un nouveau collège a été ouvert pour désengorger le collège public.

4. Nous renvoyons pour plus de détail à un article précédent publié dans L'orientation scolaire et professionnelle (Roques, 2003a).

\section{RÉSUMÉS}

Cet article propose d'étudier les rapports entre les attitudes scolaires de collégiens, interprétées en termes de projet, et le contexte spatial d'une petite ville. Si la configuration des offres locales influence les conduites juvéniles, ce travail empirique permet de mettre en évidence l'effet de la communauté environnante. En cela, les projets des jeunes ne se construisent pas essentiellement à l'intérieur de l'institution scolaire, mais en relation avec la culture des lieux. Au travers d'histoires résidentielles et de manières de représenter l'espace, on verra que le sentiment d'appartenance à la communauté locale, l'enracinement sur un territoire délimité, mais aussi la possibilité d'accéder à un lycée de la localité, influencent en grande partie la construction des projets scolaires. 
This article proposes to examine the relationship between the academic attitudes of schoolchildren, construed in terms of school projects, and the spatial context of small towns. If the configuration of local job availability influences juvenile behavior, this empirical work enables us to show the impact of the surrounding neighbourhood. Thus the school projects of the youth are not essentially shaped within the educational establishment, but in relation to local culture. Through personal residential histories, and through ways of conceiving space, one can see that the feeling of belonging to the local community, the depth of one's roots in a defined territory, with the added possibility of going to a local high school, have a major bearing on the shaping of school study plans.

\section{INDEX}

Mots-clés : Culture locale, Histoire résidentielle, Image spatiale, Projet, Territoire

Keywords : Conception of space, Local culture, Project, Residential histories, Territory

\section{AUTEUR \\ JEAN-LUC ROQUES}

est docteur en sociologie, enseignant à l'université de Montpellier III, chercheur à l'IRSA (Institut de recherche en sociologie et anthropologie), il travaille depuis plusieurs années sur les relations entre les attitudes juvéniles et la culture locale des petites villes. 